\title{
Multiscale neutronics/thermal-hyd raulics coupling with COBAYA4 code for pin-by-pin PWR transient analysis
}

\author{
N. Garcia-Herranza.*, D. Cuervo a. A. Sabatera. G. Rucabado b, S. Sanchez-Cerveraa. \\ E. Castroa
}

a Department of Energy Engineering

Universidad Politecnica de Madrid (UPM),

Jose Gutierrez Abascal, 2, 28006 Madrid,

Spain

b Centre for Computational Simulation,

UPM, Campus de Mantegancedo

Boadilla de/ Monte, 28660 Madrid, Spain

\begin{abstract}
A B S T R A C T

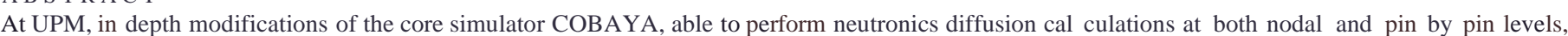

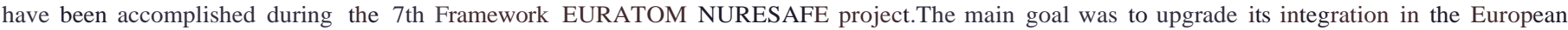

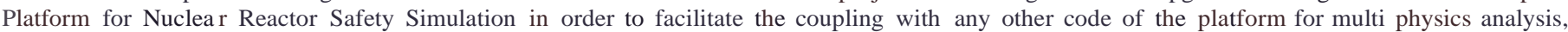

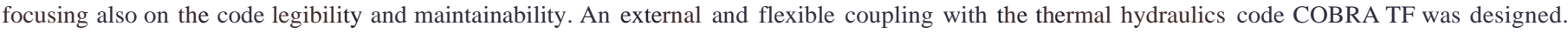

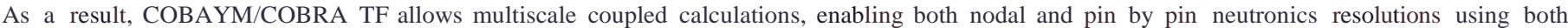

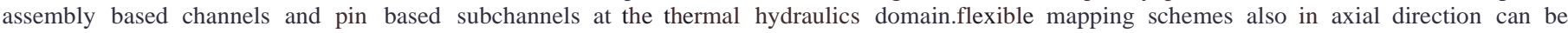

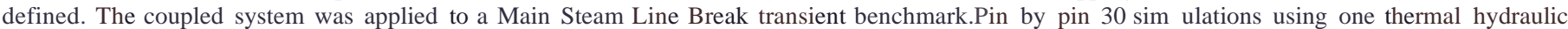

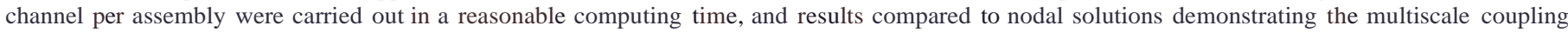

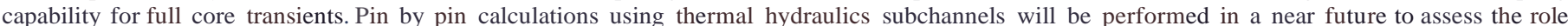

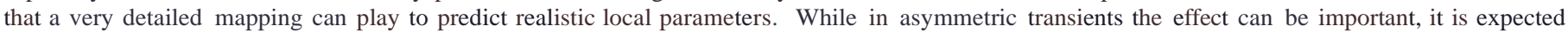

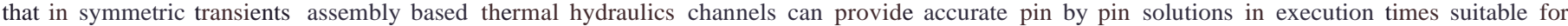

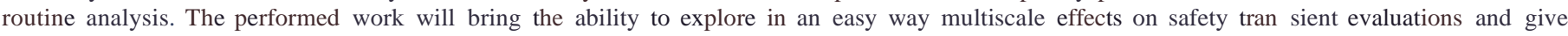
recommendations for the neutronics/thermal hydraulics mapping depending on the application
\end{abstract}

\section{Keywords:}

COBAYA/COBRA-TF

Nodal diffusion

Pin-by-pin diffusion Multi-scale

Multi-physics

Sub-channel thermal-hydraulics MSLB transient

Mapping

\section{Introduction}

Best estimate transient analysis of Light Water Reactors (LWRs) requires simulation of the relevant multiphysics phenomena occurring in the entire reactor core. High fidelity methods exist for modeling neutronics (NK), thermal hydraulics (TH) and thermal mechanics, but it is not easy to couple them in a way to be considered practical for transient analysis.

\footnotetext{
* Corresponding author

E-mail address: nuria garcia herranz@upm es (N Garcia-Herranz)
}

Different multiphysics systems are being developed to couple neutron transport codes with Computational Fluid Dynamics (CFO), sub channel or system thermal hydraulic codes. Examples of those coupled systems based on Monte Carlo transport and applied to Pressurized Water Reactor (PWR) core analysis are respectively MCNP/FLUENT (Hu and Uddin, 2008), MCNP/COBRA TF (Sanchez and Al Hamry, 2009) and Serpent/RElAPS (Wu and 
Kozlowski, 2015). Examples of coupled systems based of determin istic transport codes can be found in the Consortium for Advanced Simulation of Light Water Reactors (CASL) (Turner, 2013) and the Nuclear Energy Advanced Modeling and Simulation Program (NEAMS) Reactor Product Line (Merzari et al., 2015).

Although very active research is ongoing to develop full core neutron transport multiphysics systems, their applicability is lim ited to steady state conditions. Consequently, nuclear reactor/fuel vendors, technical support organizations and regulators rely still nowadays on standard tools for safety analysis.

Earliest standard methods used point kinetics for the neutronics core response together with thermal hydraulic system analysis codes, such as TRAC PF1 (Schnurr et al., 1992). The coupling of 3D neutron kinetics core simulators and system transient codes, such as TRACE/PARCS (Xu et al., 2006), allowed analysis of the 3D core kinetics behavior together with plant dynamics. More accurate results can be obtained if thermal hydraulic sub channel based codes, such as COBRA TF (CTF) (Salko and Avramova, 2015) are used to determine the local TH response; this is the case of global coupled code systems, such as TRAC PF1/NEM/ COBRA TF (Ziabletsev et al., 2004).

To enable those systems for safety margins' evaluations, they have to estimate the power distribution at the pin level, since var ious safety limits are based on the temperature of the hottest pel let. However, neutronic core simulations are typically carried out using nodal diffusion codes. Those codes require macroscopic cross sections homogenized over large spatial zones consisting of a fuel assembly or one quarter of the assembly. Hence only the node averaged power distribution is computed and pin power recon struction methods based on the use of assembly wise form factors (Gomez Torres et al., 2014) have to be applied in order to estimate the pin powers.

Pin by pin codes where homogenization is performed over a pin cell could be considered as the next generation of 3D core sim ulators for practical transient analysis, as the local heterogeneity effect inside the fuel assembly is partially preserved and pin wise reaction rates are available, so no approximate reconstruction methodologies have to be applied.

Pin by pin calculations are typically based on diffusion approximation or Simplified P3 approach. Codes such as SCOPE2 (Tatsumi and Yamamoto, 2003) can perform PWR full core fol low analysis using SP3 pin by pin calculations with a simplified in house thermal hydraulics module (Yamamoto and Ikeno, 2005). Also COBAYA3/COBRA TF system developed at UPM has been used to carry out 3D core pin by pin diffusion calculations for steady states using a domain decomposition technique with alternate dissections (Herrero et al., 2009). More recently, DYN SUB code system (Daeubler et al., 2015), an internal coupling of the 3D neutron kinetics code DYN3D and the sub channel code SUBCHANFLOW, was used for static and transient pin by pin simulations of the OECD NEA and U.S. NRC PWR MOX/UO2 core transient benchmark (Kozlowski and Downar, 2003). The applicability for LWR transient analysis was demonstrated, although execution times were reported to remain too high for its routine application.

The efficient coupling of pin by pin core simulators with the state of the art sub channel thermal hydraulics codes remains a challenge to provide accurate transient solutions in a reasonable time. On one hand, the generation of few group homogenized con stants at pin level is an important issue due to: $i$ ) the amount of stored data, significantly higher than at nodal level; ii) use of appropriate pin homogenization techniques to compute cross sections and discontinuity factors at the pin level, as they can be a major source of error in full core analysis.

On the other hand, Ivanov and Avramova (2007) identified, among others, the ways of coupling (internal or external) and the spatial mesh overlays as important challenges in coupling method ologies, which is especially relevant for pin by pin analysis.

The internal coupling involved in the previously mentioned pin by pin coupled systems, although more efficient, impedes an easy updating of the stand alone codes, as significant modifications are required for the coupling. However, since pin by pin calculations can be very demanding, it is desirable that the most updated ver sions of the stand alone codes (e.g. with parallel capabilities) can be used. Hence an external coupling seems the most adequate option for coupled pin by pin simulations, avoiding that mainte nance of the coupled system can require more time than the code development progress or the simulation analysis.

Also a flexible spatial coupling, where the user can specify the number of thermal hydraulic channels and neutronics nodes, is desirable. It allows developing the appropriate mapping schemes in both radial and axial directions depending on the type of transient.

At UPM, in depth modifications of the in house COBAYA diffu sion code have been accomplished during the 7th Framework EURATOM NURESAFE project (NURESAFE, 2012) in order to facili tate its integration in the NUclear REactor SIMulation (NURESIM) SALOME platform (Chanaron et al., 2015) and its coupling with any other code of the platform. An external and flexible spatial coupling with CTF code is designed, as explained in Section 2. As a result, COBAYA/CTF allows both nodal and pin by pin neutronics resolutions using both assembly based and pin based sub channel refinements at the thermal hydraulics domain. The coupled sys tem is employed in Section 3 to study the NURESAFE Main Steam Line Break (MSLB) PWR core transient benchmark at pin level and results compared to nodal solutions.

\section{Integration and coupling of COBAYA4 in SALOME platform}

\subsection{COBAYA code}

COBAYA is a multigroup neutron diffusion code for nodal and pin by pin reactor core analysis, able to compute steady state and space time kinetics problems. The first version of the code, COBAYA2 (Aragonés and Ahnert, 1986), was written in the 80's and was further developed to COBAYA3 during the 6th and 7th FP European projects NURESIM and NURISP (Chauliac et al., 2006). The last version of the code, COBAYA4, has been recoded ${ }^{\dagger}$ to be integrated in NURESIM platform including some additional capabilities.

The nodal solver employed in COBAYA (Lozano et al., 2008) is based on the Analytic Coarse Mesh Finite Difference Method (ACMFD) (Aragonés et al., 2007). It can be used as a stand alone solver or as an external acceleration for the Finite Mesh Finite Difference (FMFD) pin by pin solver. The ACMFD method combi nes a classical transverse leakage integration procedure with an analytical 1D solution of the diffusion equation on each of the spa tial directions, and can deal with 3D Cartesian and hexagonal $\mathrm{Z}$ geometries (Lozano et al., 2010). It has been extensively validated for a number of numerical benchmarks.

The pin by pin solver included in COBAYA is based on the transport corrected FMFD diffusion method. This solver can be used to solve full core problems in two different ways: using one calculation domain for the physical domain or through domain decomposition by alternate core dissections (Herrero et al., 2009). The first method is suitable in shared memory paralleliza

\footnotetext{
The code has been reformatted to FORTRAN 90, the logical control restructured to improve code readability and source maintainability, input and output subroutines redesigned to increase modularity and detect inconsistencies, compilation scheme refactored to eliminate unnecessary levels of complexity.
} 


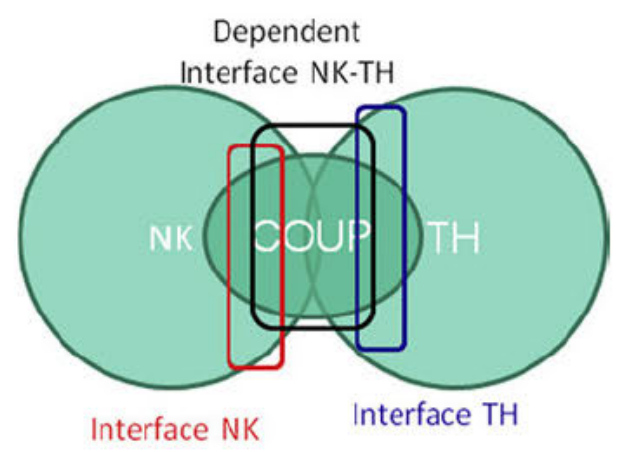

Fig. 1. Graphical description of the tight coupling.

tion and the second one is very convenient for distributed memory systems where every domain can be solved in a different machine.

COBAYA can be applied for steady state and transient calcula tions originated by control rod movement, boron concentration changes or evolutions of the coolant conditions. Transients can ini tiate from critical or subcritical conditions, requiring an initial steady state core condition that is achieved by solving an eigen value problem.

To be able to compute accurately a transient problem from a subcritical state, COBAYA includes a method to determine a virtual external neutron source (Jimenez et al., 2015). This source $S$ is intended to find a steady state solution of the subcritical system with the same flux distribution fundamental mode computed by the eigenvalue Eq. (1), where standard notation is employed ( $L$ refers to the leakage terms).

$L^{g}+\Sigma_{a}^{g} \phi^{g}+\sum_{\forall g^{\prime} \neq g} \Sigma_{s}^{g \rightarrow g^{\prime}} \phi^{g} \quad \sum_{\forall g^{\prime} \neq g} \Sigma_{s}^{g^{\prime} \rightarrow g} \phi^{g^{\prime}}+\frac{1}{k_{\text {eff }}} \chi^{g} \sum_{\forall g^{\prime}} v^{g^{\prime}} \Sigma_{f}^{g^{\prime}} \phi^{g^{\prime}}$

To achieve the steady state, the external source strength for each node and energy group is calculated as shown in Eq. (2). Using that fixed source, the transient response is computed with the standard method available in COBAYA for critical conditions.

$S^{g} \quad \frac{1 \quad k_{\text {eff }}}{k_{\text {eff }}} \chi^{g} \sum \nu^{g \prime} \Sigma^{g \prime} \phi^{g \prime}$

Recently, the capability of initiating the transient from a fixed target reactivity value has also been implemented to exactly fulfil a required Shutdown Margin (SDM). The approach consists of the following steps as proposed in (Kliem et al., 2017) by Institut de Radioprotection et de Sûreté Nucleaire (IRSN). After solving the eigenvalue equation, the ratio of the target multiplication factor to the actual $\mathrm{k}$ eff is used to correct the neutron production cross sections and the fundamental mode is recomputed. Then, the virtual external source strength is obtained as explained previously and maintained along the transient; the correction fac tor in the neutron production is also maintained to keep the reac tivity bias imposed at the initial state.

\subsection{Original state of СОВAYA coupling approach}

COBAYA3 was coupled initially to different $\mathrm{TH}$ codes in an internal or tight coupling way. This coupling was developed by FORTRAN routines that were closely connected with the control logic of each module (Fig. 1). That type of coupling was very efficient but presented two major disadvantages: it was specific for each TH code, making the structure complex, and the update to new versions was complicated. That impacted negatively the maintainability of the code system.

\subsection{Integration at NURESIM SALOME platform at nodal and pin by pin level}

The NURESIM SALOME platform, based on open source soft ware, has been developed to facilitate the coupling of codes solving the different physical phenomena in nuclear reactors, making easy the pre and post processing of numerical simulations (Chanaron et al., 2017).

The first integration of СОВАYA 3 code in the platform was per formed during NURESIM and NURISP projects only at nodal level (Jimenez et al., 2015). Pin by pin calculations were only possible outside SALOME being still based on tight coupling approach.

During NURESAFE project, it was decided to overcome the lim itations of that coupling approach and take advantage of one of the platform simulation capabilities, the medcoupling structures (SALOME6.6, 2016), for both type of calculations. Those structures allow the exchange of fields between meshes corresponding to dif ferent physics. Then, the tight coupling routines were removed keeping only the neutronics solver, which was re written in FOR TRAN 90 originating COBAYA4. It was done focusing on the future maintenance of the code structure and legibility. High Performance Computing (HPC) characteristics were also implemented through the use of a modern library of computational solvers, PETSC (Balay et al., 2015).

COBAYA4 was integrated in the NURESIM SALOME platform versions 6.6 and 7. In order to facilitate the coupling with any $\mathrm{TH}$ code inside the platform, ICoCo standard functions were implemented (Crouzet, 2015). ICoCo (Interfase COde COupling) is a set of $\mathrm{C}++$ functions defined to be used by the codes integrated in SALOME in order to execute different steps of the calculation. Those functions include the main procedures needed to carry out the simulation, such as "initialization", "solve steady state", etc. Through those ICoCo functions the code can be run in a modular way and can be coupled with other codes in the platform. A graph ical description of this new coupling approach is in Fig. 2.
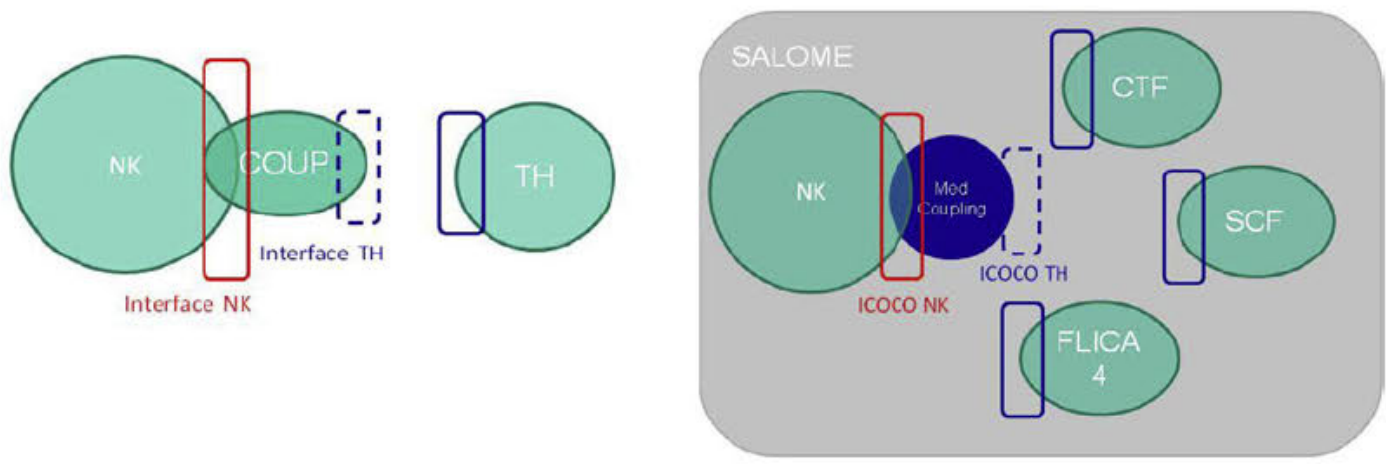

Fig. 2. Graphical description of the new coupling approach: NK and TH solvers are separated (left) and fields exchanged through medcoupling (right). 


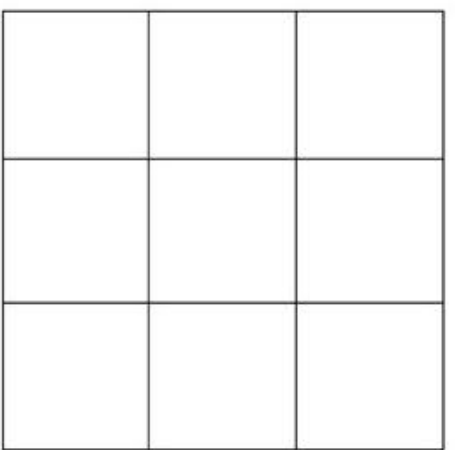

1 radial NK node per assembly COBAYA4

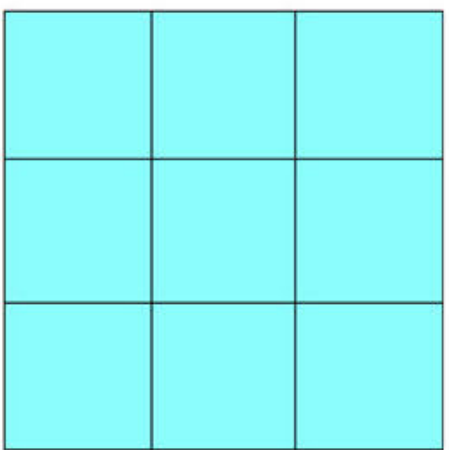

1 radial TH node per assembly (rod) CTF

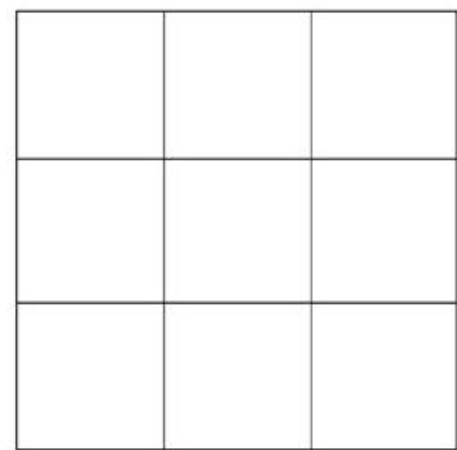

1 radial TH channel per assembly (fluid) CTF

(a) Radial meshes for NK nodal and TH channel calculations

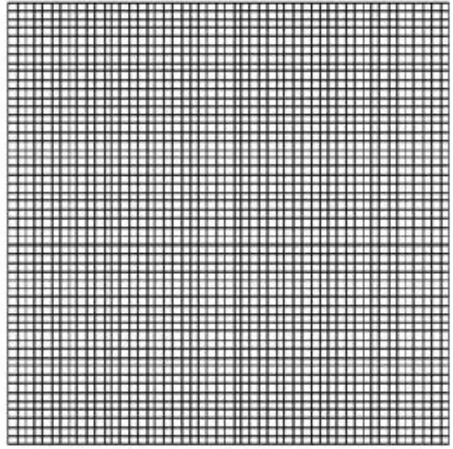

1 radial NK cell per pin COBAYA4

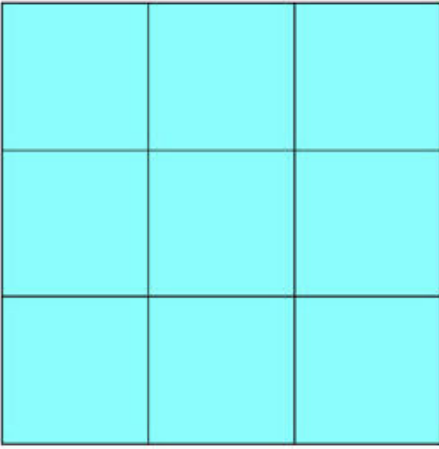

1 radial TH node per assembly (rod) CTF

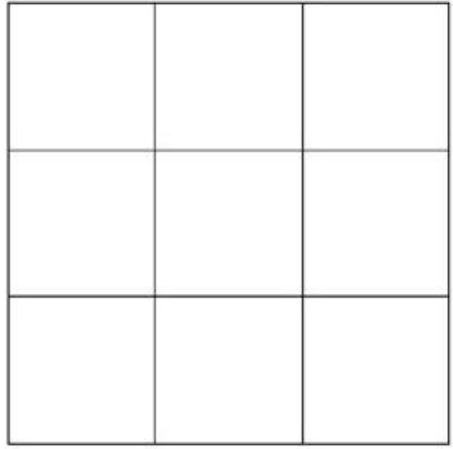

1 radial TH channel per assembly (fluid) CTF

(b) Radial meshes for NK pin-by-pin and TH channel calculations

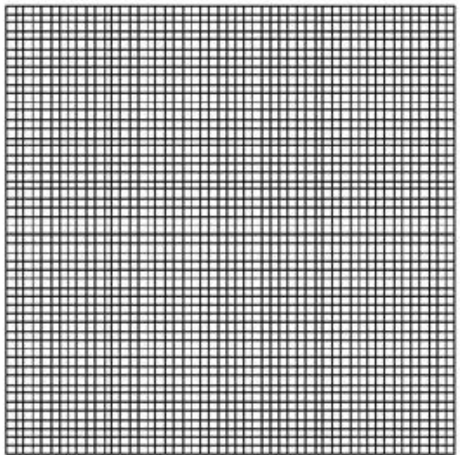

1 radial NK cell per pin COBAYA4

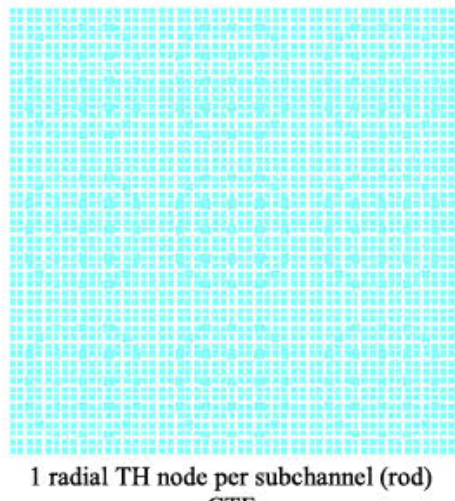
CTF

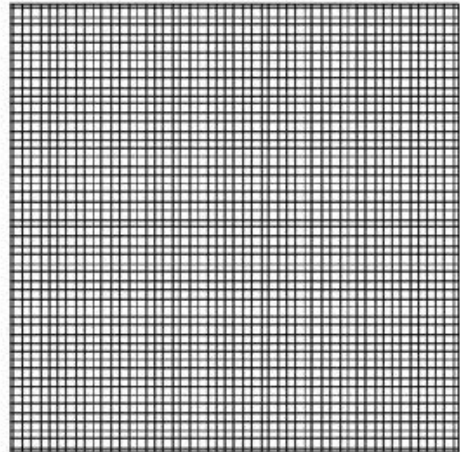

1 radial TH channel per subchannel (fluid) CTF

(c) Radial meshes for NK pin-by-pin and TH subchannel calculations

Fig. 3. Different COBAYA4 and CTF radial spatial coupling schemes in Cartesian geometry for a $3 \times 3$ assembly configuration with $17 x 17$ rods per assembly.

A mesh for each physics module has to be generated to store the fields that will be transferred from one mesh to another mesh. For COBAYA4 a Cartesian mesh generator (medHandler_CART) was designed for both pin by pin and nodal geometries. The mesher has two levels of detail: the first one defining the fuel assembly characteristics (assembly pitch, shape, etc) and the second estab lishing the sub assembly characteristics (cell pitch, shape, etc). In nodal calculations this second level represents nodes within the fuel assembly and in pin by pin calculations this level represents pin cells. In that way, the definition of the highly complex pin by pin mesh is straightforward.

The COBAYA4 component can now use the parallel structures of the SALOME platform, in order to benefit from the parallel pre and post processing capabilities of the platform. 

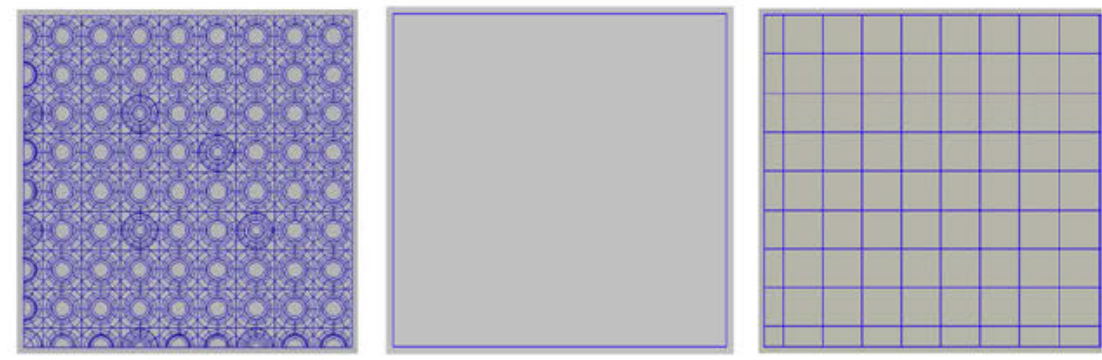

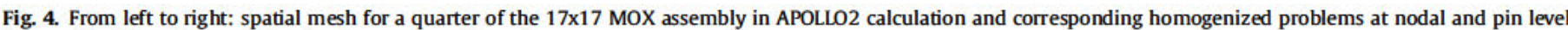
(where $\mathbf{8 1}$ different homogenized regions are considered).

Table 1

Power peaking factors at HZP steady state for pin-by-pin and nodal solutions and differences in percent with respect to the pin-by-pin solution.

\begin{tabular}{llll}
\hline & $\begin{array}{l}\text { COBAYA4/ } \\
\text { PbP }\end{array}$ & $\begin{array}{l}\text { COBAYA4/ } \\
\text { Nodal }\end{array}$ & $\begin{array}{l}\text { Diff } \\
(\%)\end{array}$ \\
\hline $\begin{array}{l}\text { Fixed multiplication factor } \\
\text { Axial power peaking factor }\left(F_{z}\right)\end{array}$ & 0.99000 & 0.99000 & \\
$\begin{array}{l}\text { Radial assembly peaking factor } \\
\quad\left(F_{x y \_a s s e m b l y}\right)\end{array}$ & 7.96 & 1.93 & $-1,2 \%$ \\
$\begin{array}{l}\text { Radial pin peaking factor }\left(F_{x y \_p i n}\right) \\
\text { Hot spot power peaking factor }\end{array}$ & 8.77 & 7.93 & $4.3 \%$ \\
$\left.\quad F_{\text {q_assembly }}\right)$ & 15.06 & - & - \\
Hot spot power peaking factor $\left.F_{\text {q_pin }}\right)$ & 17.22 & - & $2,3 \%$ \\
\hline
\end{tabular}

\subsection{Coupling with CTF code inside SALOME}

The integration in SALOME enables the possibility to couple COBAYA4 component with other codes like CTF, SUBCHANFLOW and FLICA4. In particular, it was coupled with CTF, the COBRA TF code version improved and updated by the Reactor Dynamics and Fuel Management Group of North Carolina State University.

Due to the type of integration accomplished by UPM with COBAYA4 and by Gesellschaft für Anlagen und Reaktorsicherheit (GRS) with CTF, it is possible to carry out coupled calculations using different resolutions for the NK and the TH solvers. That means that a neutronics nodal or pin by pin calculation can be performed using thermal hydraulics channels (one per assembly) or subchannels (one among four fuel rods). The interpolation tool incorporated in SALOME takes care of transferring the fields between the TH and NK meshes without having to explicitly indi cate the cells connection. It is also possible to use different types of interpolations for the physical quantities depending on the nature of the fields that are going to be transferred, delegating all the interpolation aspects in an external tool.

It is important to mention that two meshes are generated for CTF calculations. The first one is for fluid fields, like moderator den sity and temperature; the second one is for rod fields like Doppler temperature. In channel calculations those meshes coincide radi ally in space but in subchannel calculations both meshes are stag gered, being the fluid mesh coolant centered. Examples of the possible COBAYA4 neutronics meshes and the corresponding CTF fluid and rod meshes are illustrated in Fig. 3 for a $3 \times 3$ fuel assem bly configuration, with $17 \times 17$ rods per assembly.

The logical control of the COBAYA4/CTF coupling is in a python script. First, the script generates the NK and TH meshes depending on the desired level of refinement. Then, it adjusts both meshes as necessary to fit them in scale, position and angle. Finally, the script takes care of the execution of the ICoCo functions to carry out the different steps in steady state and transient calculations according to the coupling scheme established by the user. In this paper, an explicit coupling was selected. The time step for COBAYA has to be set by the user. CTF computes the TH time step as result of the flow conditions so that it is small enough to fulfill the Courant limit. If the TH time step is smaller than the neutronics one, several TH time steps are included within every NK time step.

It is worth noting that input files for the NK and TH codes are com pletely independent. The TH code can be updated to a new version with few modifications and no influence on the coupling scheme.

\section{Application to the NURESAFE PWR MSLB transient benchmark}

\subsection{Overview of the benchmark}

A challenging benchmark exercise consisting in a PWR MSLB event has been defined in the NURESAFE project. It concerns a 4 loop Westinghouse PWR Zion, with a core configuration including 193 fuel assemblies (MOX and UOX) at the end of a fuel cycle (zero boron), hot zero power conditions. The transient is initiated from a subcritical state corresponding to a $1 \%$ of SDM, All Rods In (ARI), with the highest worth control rod stuck in the overcooled area. Detailed definition of the transient can be found in (Kliem et al., 2017).

COBAYA/CTF simulations were performed inside NURESIM SAL OME platform to test the multiscale coupling during the MSLB transient. Time dependent thermal hydraulic boundary conditions were provided by Helmholtz Center Dresden Rossendorf (HZDR) using ATHLET system code to take into account the plant response.

Two transient simulations were performed: a NK nodal calcula tion (hereafter referred as Nodal) and a NK pin by pin calculation (hereafter referred as $\mathrm{PbP}$ ) using in both cases one TH channel per assembly. A pin by pin solution with TH subchannels was not computed as significant computational resources are required and MPI CTF calculations are not supported yet by the type of CTF integration inside NURESIM platform.

Hence, the CTF input deck, developed by GRS, used one TH chan nel per fuel assembly. At the active part of the channel, 30 axial nodes were defined. At the top and bottom of the channel, one node containing unheated rods was included to model the reflector.

Two COBAYA4 input decks were developed: one for the nodal calculation (with a radial node per fuel assembly) and one for the pin by pin calculation (with a radial node per pin cell). Both calculations included 34 axial nodes, 32 nodes of $11.43 \mathrm{~cm}$ for the active fuel length and two nodes for the top and bottom reflec tor. The resulting nodal mesh consists of 9826 nodes while the pin by pin mesh includes 2839714 nodes.

\subsection{Cross section libraries for coupled pin by pin calculations}

In order to compare nodal and pin by pin simulations, consis tent libraries homogenized at both nodal and pin levels were gen erated by UPM/IRSN using APOLLO2 lattice code (Sanchez et al., 1998). Two group cross sections, kinetic parameters and disconti nuity factors based on generalized equivalence theory (defined as 

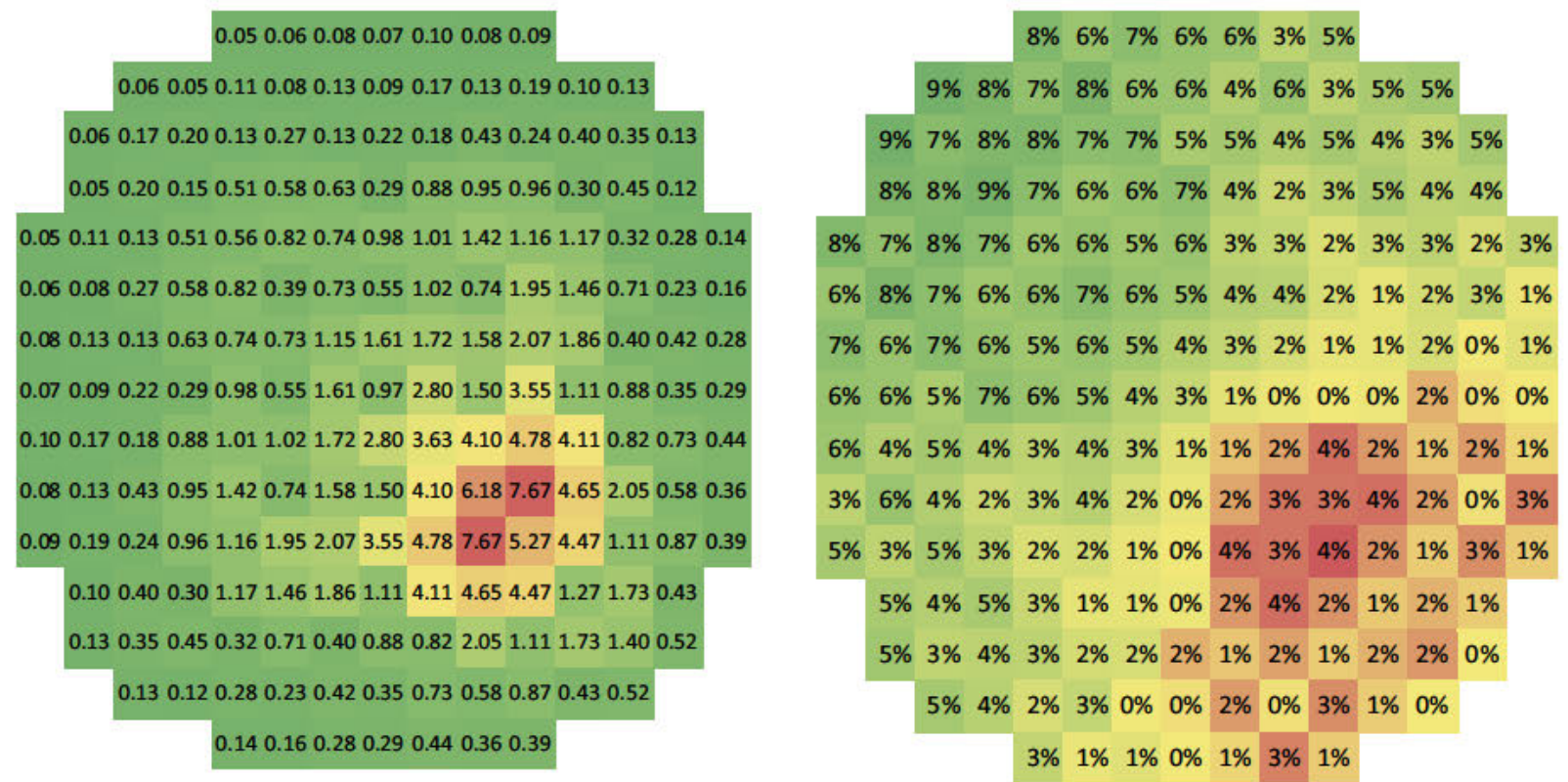

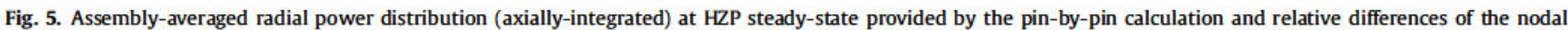
solution with respect to the pin-by-pin (diff $=($ Nodal-Pbp) $/ P b p * 100)$.

the ratio of the heterogeneous transport to homogeneous surface fluxes) were included in the form of multi dimensional tables as a function of state parameters (moderator temperature and density, fuel temperature and soluble boron concentration).

Fuel assembly calculations with reflective boundary conditions were carried out for each assembly type specified in the $3 \mathrm{D}$ core configuration map. The calculation scheme involved: first, a nom inal depletion calculation up to the assembly average burnup value given in the specifications in order to obtain the isotopic compositions; second, branch calculations to model the instanta neous effects of state variables. The actual transport flux distribu tion was used to homogenize cross sections over a node (yielding the nodal library) and over a pin cell (yielding the pin library), as displayed in Fig. 4. This way, there was no additional computational burden to compute pin wise group constants. How ever, storage requirements were increased by a factor of 81 (num ber of pins in a quarter of a $17 \times 17$ fuel assembly), since different cross section values were provided for each pin in the assembly due to their different isotopics and flux.

The APOLLO2 options used for self shielding and transport and the grid of state variables employed to build the table that com pletely encompass the range of operating conditions during the MSLB transient are presented in detail in (Kliem et al., 2017). Note that burnup is not a state variable in the library, as cross section values were computed using the isotopic compositions corre sponding to the actual burnup of each assembly in the core.

The nodal library included as assembly discontinuity factors (ADFs) the values computed by APOLLO2, as they are independent of the discretization of the low order diffusion operator used in full core calculations.

Pin discontinuity factors in the pin by pin library were com puted at each pin cell surface. In a single fuel assembly calculation, pin cells have non zero currents at boundaries, so discontinuity factors must be calculated according to the diffusion operator dis cretization that will be used in COBAYA. Then, a routine was added to the post processing tool of APOLLO outputs, to use the transport average flux and net currents at the boundary of each pin to com pute the homogeneous interface fluxes using COBAYA FMFD dis cretization. Interface discontinuity factors were computed at each boundary as the ratio of the heterogeneous to the homoge neous interface fluxes.

Using zero leakage pin cell calculations to produce pin wise homogenized values was discarded because non linear corrections accounting for the pin environment would be required in an itera tive process during the core diffusion calculation (Herrero et al., 2012).

Reflector cross sections were generated by IRSN using Lefebvre Lebigot method (Marguet, 2011), based on a one dimensional model of the reflected reactor. The same constants (no discontinuity factors were provided) were used for nodal and pin by pin calculations.

\subsection{Results}

\subsubsection{HZP steady state}

The initial state of the reactor core before the MSLB event has been computed. A fixed value of 0.99 for the effective multiplica tion factor was set following the specifications.

A summary of results is in Table 1. Comparisons of the power distributions provided by the pin by pin and nodal calculations are in Figs. 5 and 6 . It can be seen that the power profile is radially shifted to the core area with the withdrawn rod. The maximum discrepancies in the radial power distribution between both calcu lations appear at the diametrically opposite zone. The pin by pin solution predicts a radial assembly peaking factor $\mathrm{Fxy}^{\S} \sim 4 \%$ lower than the nodal solution, showing that the fine mesh calculation slightly flattens the assembly averaged radial power. The radial pin peaking factor is $14 \%$ higher than the radial assembly peaking factor suggesting a strong peaked distribution in the hottest assembly, information that is lost in the calculation at nodal level (since no reconstruction technique is used in COBAYA).

The axial peaking factor $\mathrm{Fz}$ is similar in both simulations (both are using the same axial mesh), 1\% higher in the pin by pin case. That is, the axial power profile is not very sensitive to the radial

\footnotetext{
$\S$ Radial peaking factor $F x y$ is defined in this paper as the ratio of the maximum axially-integrated rod power within the core to the average rod power. If the power is averaged at assembly level, the corresponding factor is referred as radial assembly peaking factor.
} 

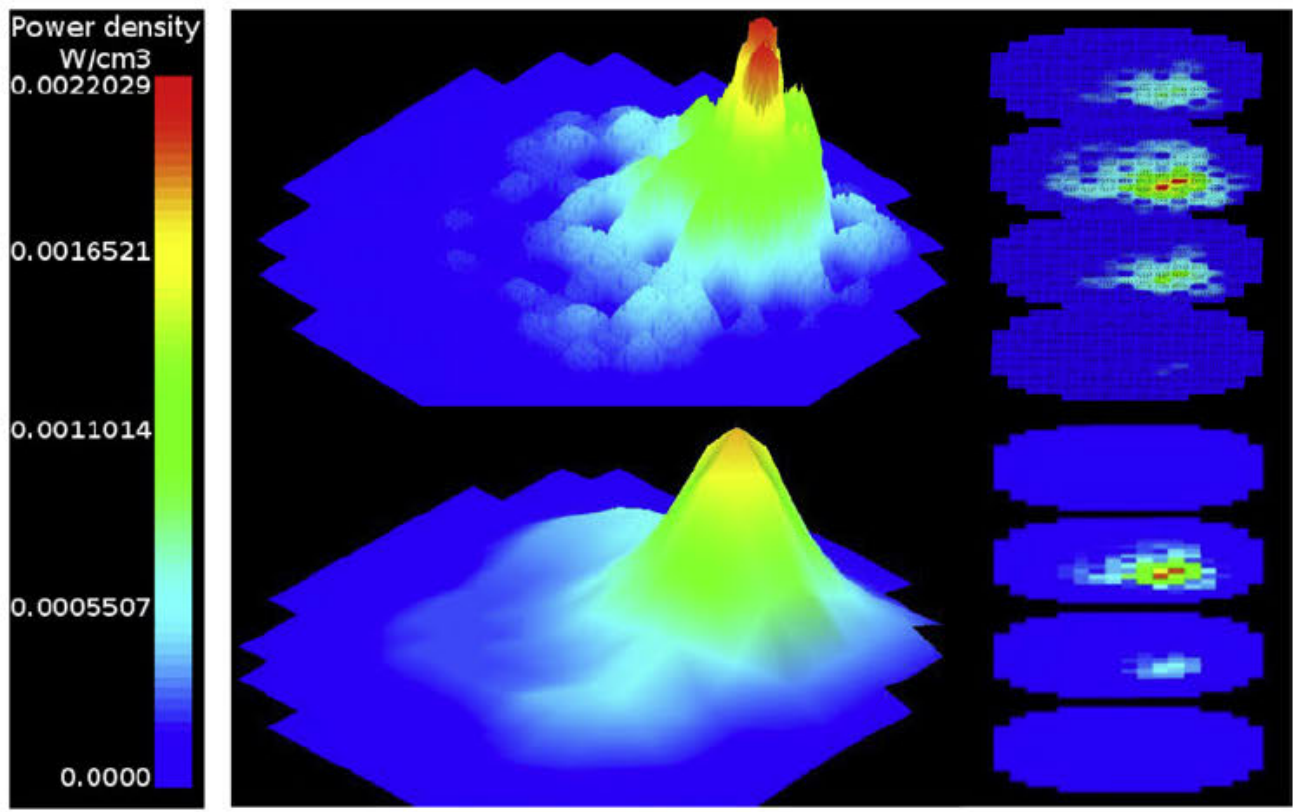

Fig. 6. Power density distributions at HZP steady-state computed by pin-by-pin and nodal calculations post-processed by SALOME6.6.
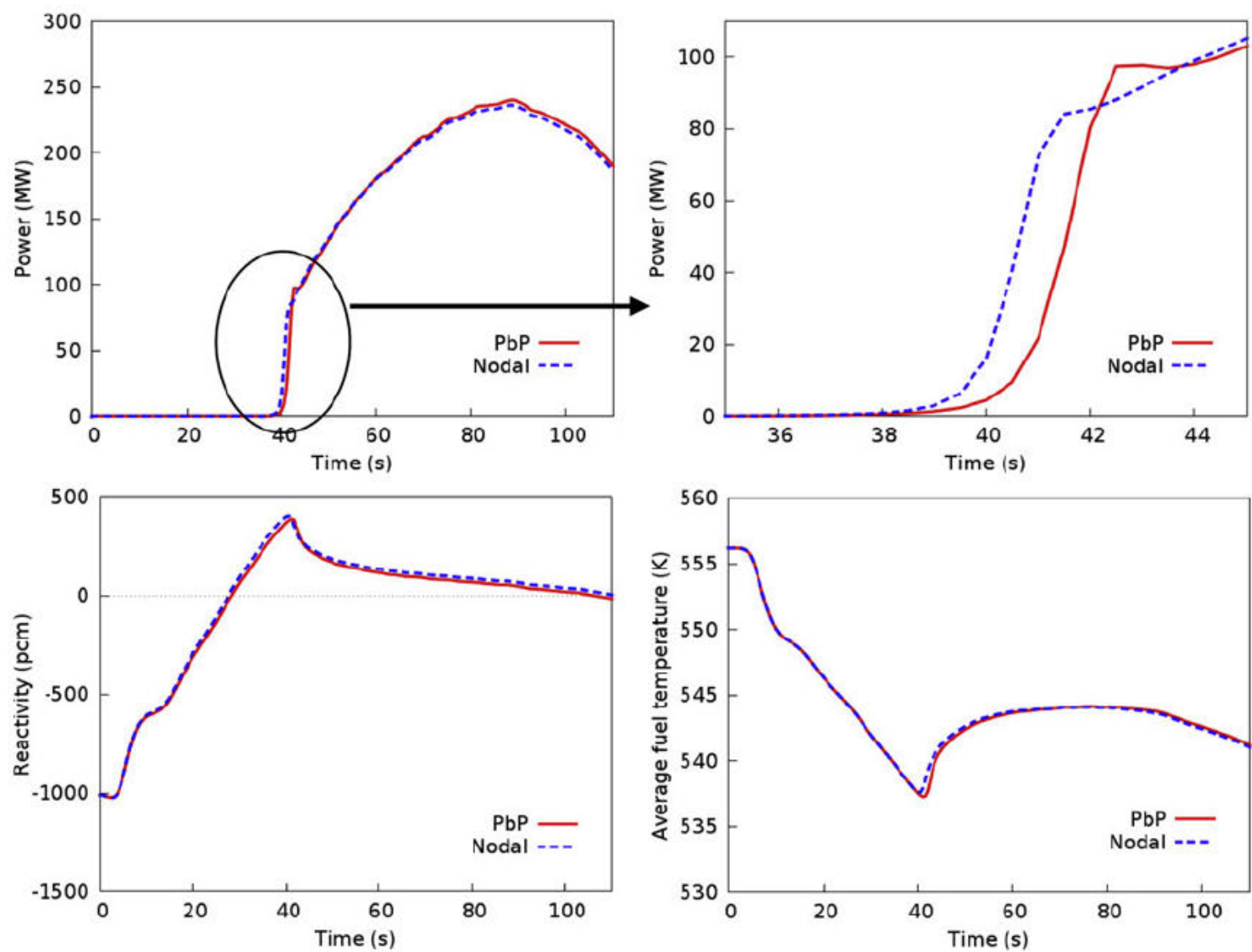

Fig. 7. Core reactor power, reactivity and average fuel temperature during the transient for pin-by-pin and nodal calculations.

spatial meshing. Concerning the total or hot spot peaking factor $\mathrm{Fq}^{* *}$ the pin by pin solution provides a value $\sim 2 \%$ lower than the

\footnotetext{
* Total or hot stop peaking factor $F q$ is defined as the ratio of the maximum power density within the core to the average core power density.
}

nodal solution, reflecting again the influence of the radial mapping on the peaking factors conditioned by the radial power profile.

SALOME6.6 post processor tool was used to generate the 3D visualization of the power distributions shown in Fig. 6.

The steady state pin by pin calculation took around $3 \mathrm{~h}$ on a single Intel Core i7 at $3.07 \mathrm{GHz}$, while the steady state nodal 
Table 2

Comparison of parameters corresponding to the main transient events.

\begin{tabular}{|c|c|c|c|c|}
\hline & \multicolumn{2}{|c|}{ COBAYA4/PbP } & \multicolumn{2}{|c|}{ COBAYA4/Nodal } \\
\hline & Time (s) & Value & Time (s) & Value \\
\hline Core criticality & 28.5 & - & 28 & - \\
\hline Maximum reactivity & 41 & $387.6 \mathrm{pcm}$ & 40.5 & $403.0 \mathrm{pcm}$ \\
\hline Maximum core power & 89 & $240.3 \mathrm{MW}$ & 88.5 & $236.6 \mathrm{MW}$ \\
\hline Return to subcriticality & 106.5 & - & 111 & - \\
\hline
\end{tabular}
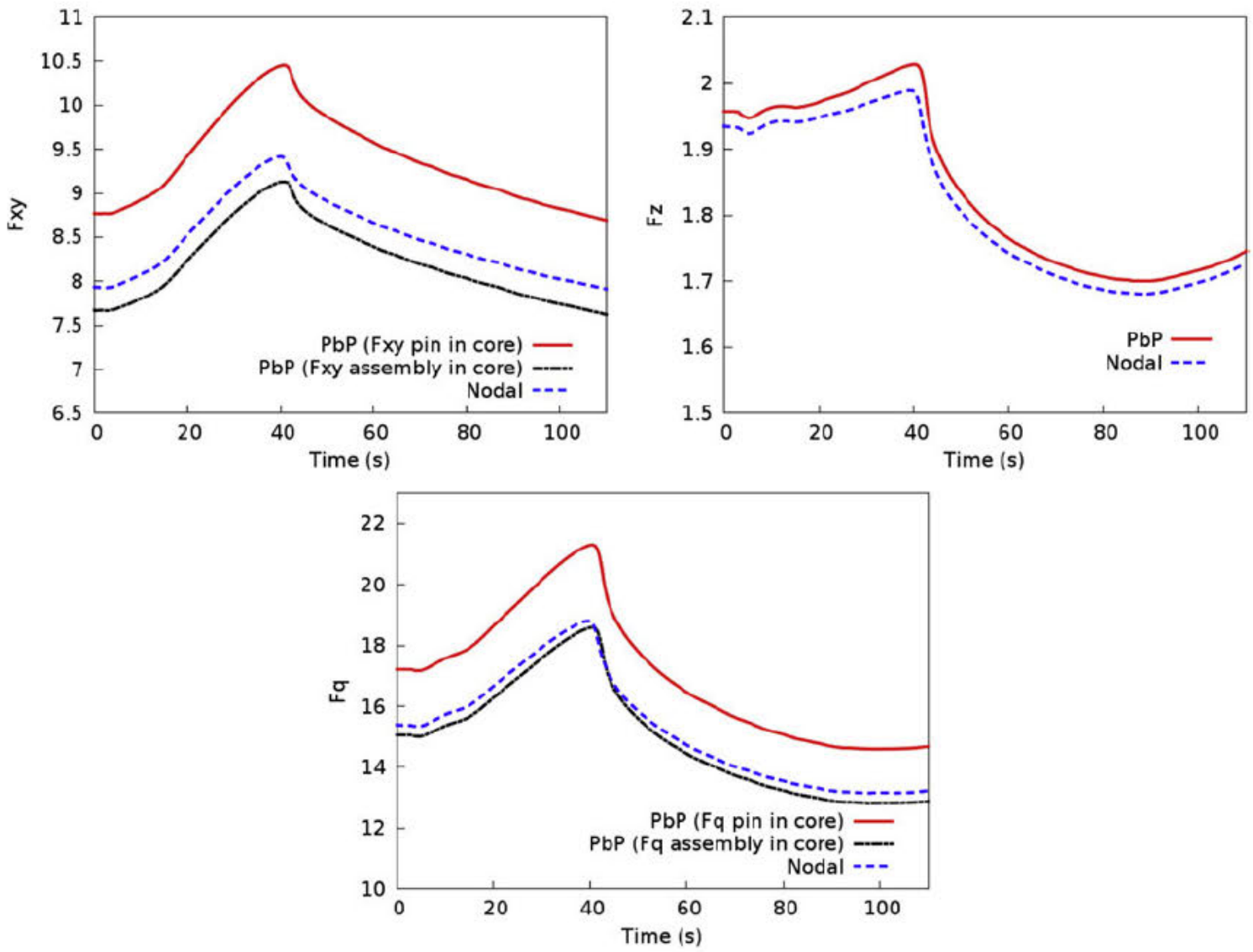

Fig. 8. Evolution of the radial peaking factor Fxy, axial peaking factor Fz and hot spot peaking factor Fq along the transient for pin-by-pin and nodal calculations.

calculation took around $3 \mathrm{~min}$. on the same core. In the pin by pin calculation, $87 \%$ of the overall run time was consumed by the mesh generator/interpolator, $11 \%$ by COBAYA 4 and $2 \%$ by CTF. In the nodal calculation, the mesh generator/interpolator was responsible for $49 \%$ of the run time, COBAYA4 for $12 \%$ and CTF for $39 \%$. The mesh generator/interpolator constructs the NK and TH nodes on the geometry, establishes the nodal connectivity and returns the interpolation matrix to exchange the feedback variables between the NK and TH meshes. As expected, this process for the pin by pin case is time consuming and represents a major cost in the steady state simulation.

\subsubsection{Transient}

The evolution of core parameters during the transient is shown in Fig. 7. Pin by pin and nodal simulations provide similar global results; comparisons of relevant parameters characterizing the main events during the transient are in Table 2.

After the overcooling caused by the MSLB event (occurring at $0 \mathrm{~s}$ ), there is a positive reactivity insertion. The core criticality is reached at $\sim 28 \mathrm{~s}$ and maximum reactivity at $\sim 41 \mathrm{~s}$, when a power excursion occurs further followed by a smooth power rise. Prompt
Table 3

Peaking factors at the moment of maximum core power and differences in percent with respect to the pin-by-pin solution.

\begin{tabular}{llll}
\hline & $\begin{array}{l}\text { COBAYA4/ } \\
\text { PbP }\end{array}$ & $\begin{array}{l}\text { COBAYA4/ } \\
\text { Nodal }\end{array}$ & $\begin{array}{l}\text { Diff } \\
(\%)\end{array}$ \\
\hline $\begin{array}{l}\text { Axial power peaking factor }\left(F_{z}\right) \\
\text { Radial assembly peaking factor }\end{array}$ & 1.70 & 1.68 & $-1.1 \%$ \\
$\quad\left(F_{x y \_a s s e m b i y}\right)$ & 7.89 & 8.18 & $3.6 \%$ \\
$\begin{array}{l}\text { Radial pin peaking factor }\left(F_{x y \_p i n}\right) \\
\text { Hot spot power peaking factor }\end{array}$ & 9.00 & - & - \\
$\left.\quad F_{\text {q_assembly }}\right)$ & 12.93 & 13.27 & $2.6 \%$ \\
Hot spot power peaking factor $\left.F_{q \_p i n}\right)$ & 14.72 & - & - \\
\hline
\end{tabular}

criticality is never attained as reactivity keeps lower than $1 \$$ (the beta delayed neutron fraction is $482 \mathrm{pcm}$ ). The initial power excur sion in the pin by pin solution is delayed with respect to the nodal solution by around $1 \mathrm{~s}$. The maximum level of core power was 240.3 MW, 1.7\% higher than for the nodal solution, in both cases $\sim 89$ s after the MSLB event. The increase in the fuel temperature after the power rise leads to a negative reactivity insertion due to the Doppler feedback effect so that the core reactivity decreases progressively. Slight differences can be appreciated between the 

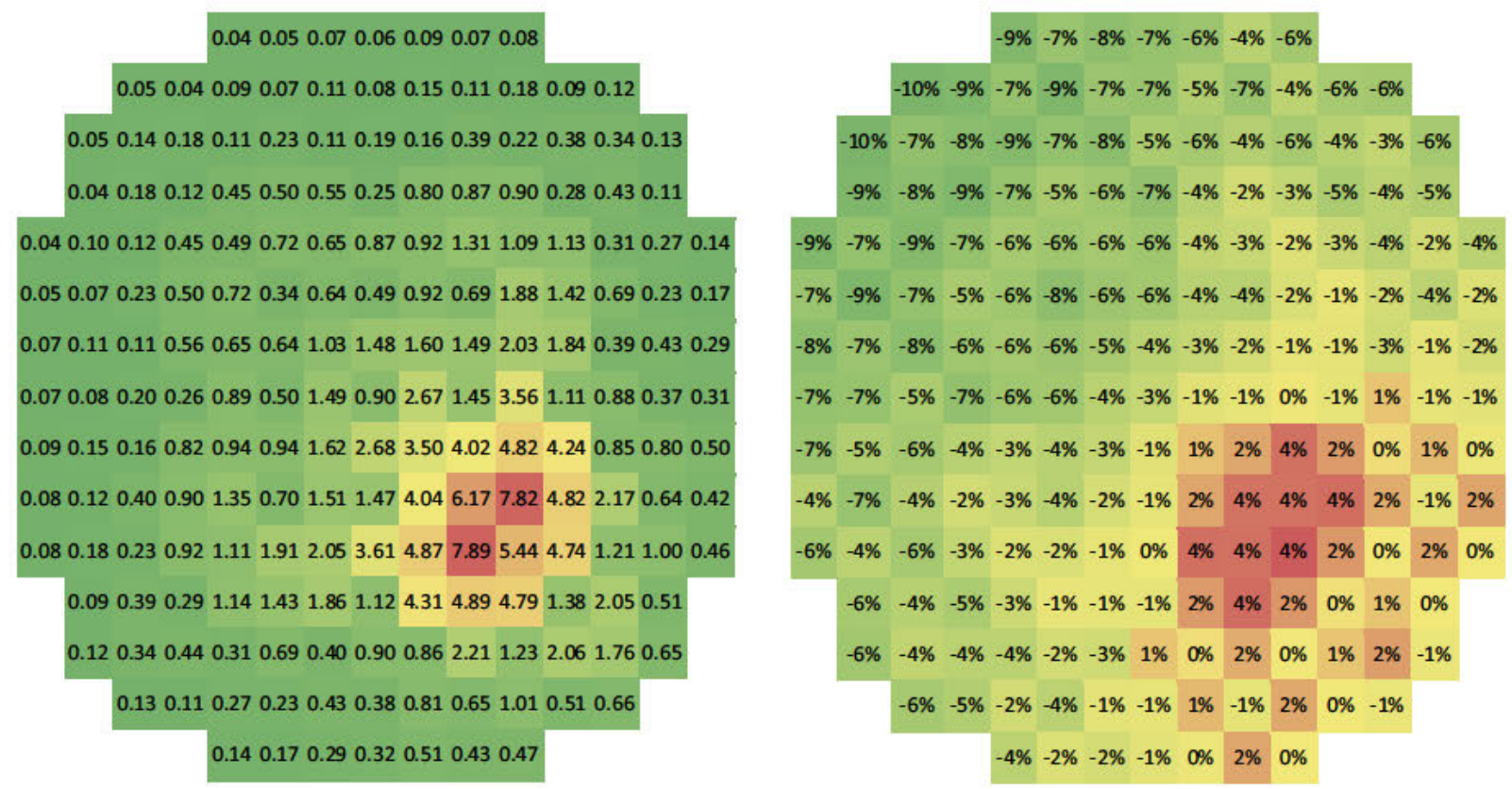

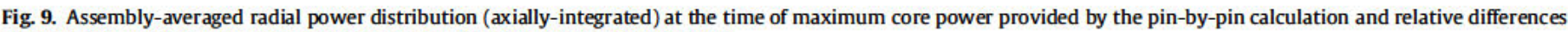
of the nodal solution with respect to the pin-by-pin (diff $=($ Nodal-Pbp)/Pbp*100).

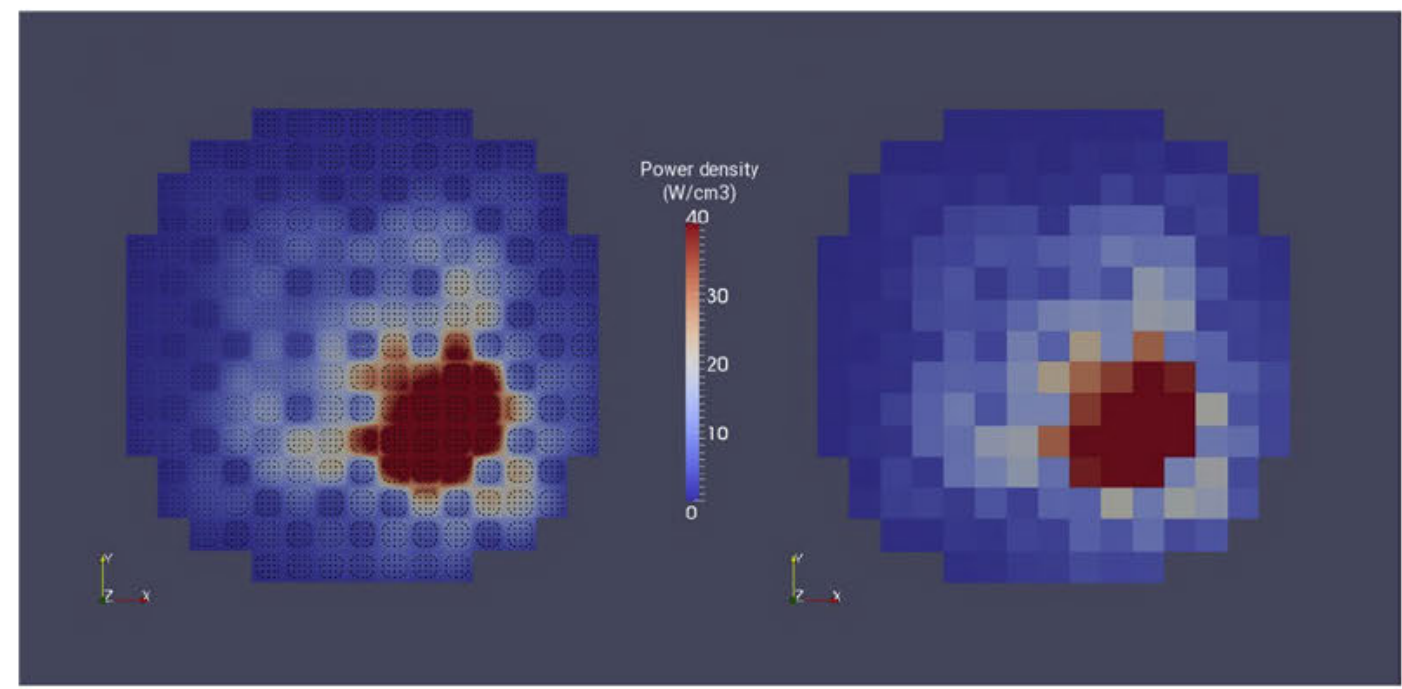

Fig. 10. Radial power distribution (axially-integrated) for the pin-by-pin (at the pin level) and the nodal calculation at the time of maximum core power.

pin by pin and nodal solutions due to the different power feedback in both simulations. Note that, even if CTF uses in both cases one radial TH node per assembly to compute the fuel temperature, the assembly power transferred by COBAYA4 differs between a pin by pin and a nodal calculation.

The evolution of the power peaking factors along the transient is shown in Fig. 8. The same trend mentioned in the steady state is observed, that is, the pin by pin solution predicts a radial assem bly peaking factor Fxy and a hot spot peaking factor Fq lower than the nodal solution, being the axial peaking factors Fz quite similar.

At the moment of maximum core power, peaking factors values are illustrated in Table 3 and a detailed comparison of the assembly averaged radial power profile is shown in Fig. 9. It can be seen that the pin by pin simulation provides an assembly averaged power distribution slightly more flattened than the nodal simulation. Fig. 10 shows the radial power density obtained with
SALOME6.6 visualization tool, where the pin resolution can be observed.

Pin power peaking factors (Fxy and Fq) are around 14\% higher than the corresponding assembly power peaks, indicating that the gradients in the hottest assembly are similar to the steady state con dition. It should be taken into account that in the pin by pin calcu lation subchannel effects are not considered, so the local heterogeneity in thermal hydraulics model inside the assembly is lost and an average of the TH fields are used during the pin wise cross sections feedback. As a result, the power distribution within the fuel assemblies could be less peaked if a subchannel calculation was used.

The pin by pin transient calculation took about $50 \mathrm{~h}$ on a single Intel Core i7 at $3.07 \mathrm{GHz}$, while the nodal transient calculation took about $40 \mathrm{~min}$. on the same machine. This computational cost is reasonable for $3 \mathrm{D}$ pin by pin safety analysis. 


\section{Conclusions}

New developments in the multi group neutron diffusion COBAYA code have enabled its efficient integration into the NURE SIM SALOME platform. After the coupling with the thermal hydraulics code CTF, it is possible to carry out coupled calculations inside the platform using different resolutions for the neutronics and the thermal hydraulic solvers. That is, nodal or pin by pin transport corrected diffusion calculations can be performed using channel or subchannel models, bringing the ability to explore mul tiscale effects on safety transient analysis.

The coupled system has been applied to the MSLB PWR tran sient benchmark defined in the frame of NURESAFE project. Pin by pin and nodal solutions were computed using in both cases assembly based thermal hydraulic channels. Pin wise homoge nized constants, including pin discontinuity factors, fully consis tent with the constants homogenized at nodal level were generated using APOLLO2 transport code.

The pin by pin calculation was carried out in a reasonable com puting time. While global parameters such as the total power or reactivity along the transient were not very sensitive to the neu tronics scale in the multiphysics simulation, differences up to $4 \%$ between calculations at pin and nodal level were found in assem bly peaking factors conditioned by the radial power profile. Pin peaking factors reflected strong gradients within the hottest assembly. Taking into account that local feedback effects are not modeled, it is possible that the pin by pin calculation could be overestimating local peak powers.

Pin by pin calculations using thermal hydraulics subchannels will be performed in a near future to assess the role that a very detailed mapping can play for realistic local parameters predic tions. While in this kind of spatial asymmetric transients the effect can be important, it is expected that in other transients the much faster employed N/TH spatial coupling scheme could provide accu rate pin by pin solutions, making 3D pin by pin tools better candi dates than nodal methods for routine safety applications.

As the main objective was to test the capability of the multi scale coupling, no special attention was paid to different aspects that impact accuracy such as axial nodalization in the pin by pin calculation, time steps in the coupling algorithm, etc. Hence results should not be considered as definitive in terms of accuracy, but as a proof that pin based results can differ from assembly based predictions, stressing the importance of pin by pin analysis for local safety evaluations.

Validation of the coupled system with respect to MCNP/CTF is ongoing in collaboration with North State Carolina University. Fur ther investigations will be then performed to explore the multiscale effects on safety transient evaluations and give recommendations for the neutronics/thermal hydraulics mapping depending on the application.

\section{Acknowledgements}

This work was funded by the EU Commission under the 7th EURATOM Framework Program, within the Collaborative Project NURESAFE under contract 323263. Authors acknowledge the com puter resources and technical assistance provided by Centro de Supercomputación y Visualización de Madrid (CeSViMa) as well as GRS for the work performed on integration of CTF in SALOME platform.

\section{References}

Aragonés, J.M., Ahnert, C., 1986. A linear discontinuous finite difference formulation for synthetic coarse-mesh few-group diffusion calculations. Nucl. Sci. Eng. 94 (4), 309-322.
Aragonés, J.M., Ahnert, C., García-Herranz, N., 2007. The analytic coarse-mesh finite difference method for multigroup and multidimensional diffusion calculations. Nuc. Sci. Eng. 157, 1-15.

Balay, S., Abhyankar, S., Adams, M. F., Brown, J., Brune, P., Buschelman, K., Dalcin, L., Eijkhout, V., Gropp, W. D., Kaushik, D., Knepley, M. G., McInnes, L. C., Rupp, K., Smith, B. F., Zampini, S., Zhang, H., 2015. PETSc Users Manual. ANL-95/11 Revision 3.6. Argonne National Laboratory.

Chanaron, B., Ahnert, C., Crouzet, N., Sanchez, V., Kolev, N., Marchand, O., Kliem, S., Papukchiev, A., 2015. Advanced multi-physics simulation for reactor safety in the framework of the NURESAFE project. Ann. Nucl. Energy 84, 166-177.

Chanaron, B., Kliem, S., Bestion, D., Lakehal, D., Crouzet, N., 2017. Overview of the NURESAFE European Project. Nucl. Eng, Dsgn. submitted.

Chauliac, C., Aragonés, J. M., Bestion, D., Cacuci, D. G., Coddington, P., Dada, L., 2006. NURESIM: A European Platform for Nuclear Reactor Simulation. ICONE-14. Workshop on advanced LWR, Miami, USA.

Crouzet, N., 2015. NURESAFE Internal Report D41.21: Report on the update of the mesh and field structures in the coupled codes.

Daeubler, M., Trost, N., Jimenez, J., Sanchez, V., Stieglitz, R., Macian-Juan, R., 2015. Static and transient pin-by-pin simulations of a full PWR core with the extended coupled code system DYNSUB. Ann. Nucl. Energy 84, 31-44.

Gomez-Torres, A.M., Sanchez, V., Kliem, S., Gommlich, A., 2014. Implementation of a fast running full core pin power reconstruction method in DYN3D. Nucl. Eng, Dsgn. 274, 44-55.

Herrero, J.J., García-Herranz, N., Cuervo, D., Ahnert, C., 2012. Neighborhoodcorrected interface discontinuity factors for multi-group pin-by-pin diffusion calculations for LWR. Ann. Nucl. Energy 46, 106-115.

Herrero, J.J., Jiménez, J., Aragonés, J.M., Ahnert, C., 2009. Performance of whole core pin-by-pin calculations by domain decomposition through alternate dissections in steady state and transient calculations. In: Proceedings of 2009 International Conference on Mathematics, Computational Methods and Reactor Physics (M\&C 2009), May. Saratoga Springs, New York, pp. 3-7.

Hu, J., Uddin, R., 2008. Coupled neutronics and thermal-hydraulics using MCNP and FLUENT. Trans. Am. Nucl. Soc. 98, 606-608.

Ivanov, K., Avramova, M., 2007. Challenges in coupled thermal-hydraulics and neutronics simulations for LWR safety analysis. Ann. Nucl. Energy 34 (6), 501-513.

Jimenez, G., Herrero, J.J., Gommlich, A., Kliem, S., Cuervo, D., Jimenez, J., 2015. Boron dilution transient simulation analyses in a PWR with neutronics/thermalhydraulics coupled codes in the NURISP project. Ann. Nucl. Energy 84, 86-97.

Kliem, S., Kozmenkov, Y., Hadek, J., Perin, Y., Fouquet, F., Bernard, F., Sargeni, A., Cuervo, D., Sabater, A., Sanchez-Cervera, S., Garcia-Herranz, N., Zerkak, O., Ferroukhi, H., Mala, P., 2017. Testing the NURESIM platform on a PWR main steam line break benchmark. Nucl. Eng, Dsgn., 321, 8-25.

Kozlowski, T., Downar, T.J., 2003. OECD/NEA and US NRC PWR MOX/UO2 core transient benchmark. NEA/NSC/DOC(2003) 20. Working Party of the Physics of Plutonium Fuels and Innovative Fuel Cycles, OECD/NEA Nuclear Science Committee.

Lozano, J.A., García-Herranz, N., Ahnert, C., Aragonés, J.M., 2008. The analytic nodal diffusion solver ANDES in multigroups for 3D rectangular geometry: development and performance analysis. Ann. Nucl. Energy 35 (12), 2365-2374.

Lozano, J.A., Jiménez, J., García-Herranz, N., Aragonés, J.M., 2010. Extension of the analytic nodal diffusion solver ANDES to triangular-Z geometry and coupling with COBRA-IIIc for hexagonal core analysis. Ann. Nucl. Energy 37 (3), 380-388.

Marguet, S. La physique des réacteurs nucléaires. Collection EDF R\&D, Editions Lavoisier Tec \& Doc, ISBN 978-2-7430-1105-5 (2011).

NURESAFE, 2012, Nuclear Reactor Safety Simulation Platform, Description of work, FP7-Fission.

Merzari, E., Shemon, E., Yu, Y., Thomas, J., Obabko, A., Jain, R., Mahadevan, V., Solberg, J., Ferencz, R., Whitesides, R., 2015 Full Core Multi-Physics Simulation with Offline Core Deformation, Argonne National Laboratory Report ANL/NE-15/42.

Salko, R., Avramova, M., 2015. COBRA-TF Subchannel Thermal-Hydraulics Code (CTF) Theory Manual, CASL-U-2015-0054-000.

SALOME6.6 Documentation (2016), www.salome-platform.org. (visited 01/12/2016).

Sanchez, R., Mondot, J., Stankovski, Z., Cossic, A., Zmijarevic, I., 1998. APOLLO II: a user-oriented, portable, modular code for multigroup transport assembly calculations. Nuc. Sci. Eng. 100 (3), 352-362.

Sanchez, V., Al-Hamry, A., 2009. Development of a coupling scheme between MCNP and COBRA-TF for the prediction of the pin power of a PWR fuel assembly. In: Proceedings of 2009 International Conference on Mathematics, Computational Methods and Reactor Physics (M\&C 2009), May. Saratoga Springs, New York, pp. 3-7.

Schnurr, N.M., Steinke, R.G., Martinez, V., Spore, J.W., 1992. TRAC-PF1/MOD2 code manual, NUREG/CR-5673, LA-12031-M. vol. 2.

Tatsumi, M., Yamamoto, A., 2003. Advanced PWR core calculation based on multigroup nodal-transport method in three-dimensional pin-by-pin geometry. J. Nucl. Sci. Technol. 40 (6), 376-387.

Turner, J., 2013, Virtual Environment for Reactor Applications (VERA): Snapshot 3.1, CASL Technical Report: CASL-U-2013-0164-000.

Wu, X., Kozlowski, T., 2015. Coupling of system thermal-hydraulics and MonteCarlo code: convergence criteria and quantification of correlation between statistical uncertainty and coupled error. Ann. Nucl. Energy 75, 377-387.

Xu, Y., Downar, T., Ward, A., Kozlowski, T., Ivanov, K., 2006. Multi-physics coupled code reactor analysis with the U.S. NRC code system TRACE/PARCS. In: Proceedings of PHYSOR-2006 Conference, CD-ROM, Vancouver, Canada.

Yamamoto, A., Ikeno, T., 2005. Impact of pin-by-pin thermal-hydraulic feedback modeling on steady-state core characteristics. Nucl. Technol. 149 (2), 175-188.

Ziabletsev, D., Avramova, M., Ivanov, K., 2004. Development of Pressurized Water Reactor integrated safety analysis methodology using multilevel coupling algorithm. Nuc. Sci. Eng. 148 (3), 414-425. 